\title{
Generalized factor analyses for contingency tables
}

François Bavaud

Section d'Informatique et de Méthodes Mathématiques, Faculté des Lettres, Université de Lausanne

CH-1015 Lausanne-Dorigny

(e-mail: Francois.Bavaud@imm.unil.ch)

Summary. Quotient dissimilarities constitute a broad aggregation-invariant family; among them, $f$-dissimilarities are Euclidean embeddable (Bavaud 2002). We present a non linear principal components analysis (NPA) applicable to any quotient dissimilarity, based upon the spectral decomposition of the central inertia. For $f$-dissimilarities, the same decomposition yields a non linear correspondence analysis (NCA), permitting to modulate as finely as wished the contributions of positive or negative deviations from independence. The resulting coordinates exactly reproduce the original dissimilarities between rows or between columns; however, Huygens's weak principle is generally violated, as measured by some quantity we call eccentricity.

\section{Introduction and notations}

Let $n_{j k}$ be a $(J \times K)$ contingency table, with relative frequencies $f_{j k}:=n_{j k} / n$. Marginal profiles (assumed strictly positive) are $\rho_{j}^{*}:=n_{j} \bullet / n=f_{j} \bullet$ and $\rho_{k}:=$ $n_{\bullet k} / n=f_{\bullet k}$, where $n_{j \bullet}:=\sum_{k \in K} n_{j k}$ are the row marginals, $n_{\bullet k}:=\sum_{j \in J} n_{j k}$ are the column marginals, and $n:=n_{\bullet \bullet}$ is the grand total. The independence quotients $q_{j k}$ are the ratios of the observed counts to the expected counts under independence:

$q_{j k}:=\frac{n_{j k} n}{n_{j \bullet} n_{\bullet k}}=\frac{f_{j k}}{\rho_{j}^{*} \rho_{k}} \quad$ with $\quad \sum_{j \in J} \rho_{j}^{*} q_{j k}=1 \quad \forall k \quad \sum_{k \in K} \rho_{k} q_{j k}=1 \quad \forall j$.

One has $q_{j k}=1$ for all cells iff perfect independence holds. The chi-square dissimilarity $D_{j j^{\prime}}^{\chi}$ between rows $j$ and $j^{\prime}$ expresses in terms of quotients as $D_{j j^{\prime}}^{\chi}=\sum_{k} \rho_{k}\left(q_{j k}-q_{j^{\prime} k}\right)^{2}$.

Quotient dissimilarities are of the form $D_{j j^{\prime}}:=\sum_{k} \rho_{k} F\left(q_{j k}, q_{j^{\prime} k}\right)$, where $F\left(q, q^{\prime}\right) \geq 0, F\left(q, q^{\prime}\right)=F\left(q^{\prime}, q\right)$ and $F(q, q)=0 ; f$-dissimilarities are quotient dissimilarities with $F\left(q, q^{\prime}\right)=\left(f(q)-f\left(q^{\prime}\right)\right)^{2}$, whereas $g$-dissimilarities are 
quotient dissimilarities with $F\left(q, q^{\prime}\right)=\left(g(q)-g\left(q^{\prime}\right)\right)\left(q-q^{\prime}\right)$ (Bavaud 2002). Quotient dissimilarities are aggregation-invariant, $f$-dissimilarities are $(\mathrm{Eu}-$ clidean) embeddable, that is representable as $D_{j j^{\prime}}=\sum_{l}\left(x_{j l}-x_{j^{\prime} l}\right)^{2}$ (where $x_{j l}$ is the coordinate of object $j$ in dimension $l$ ), and $g$-dissimilarities obey Huygens's weak principle $I_{1}=I_{2}$ where $I_{1}:=\sum_{j} \rho_{j}^{*} D_{j g}$ is the central inertia $^{1}$ and $I_{2}:=\frac{1}{2} \sum_{j j^{\prime}} \rho_{j}^{*} \rho_{j^{\prime}}^{*} D_{j j^{\prime}}$ the pair inertia (Bavaud 2002); moreover, the only member common to both families is (up to a constant) the chi-square dissimilarity characterized by $F^{\chi}\left(q \cdot q^{\prime}\right)=\left(a-a^{\prime}\right)^{2}$ (see Figure 1$)^{2}$.

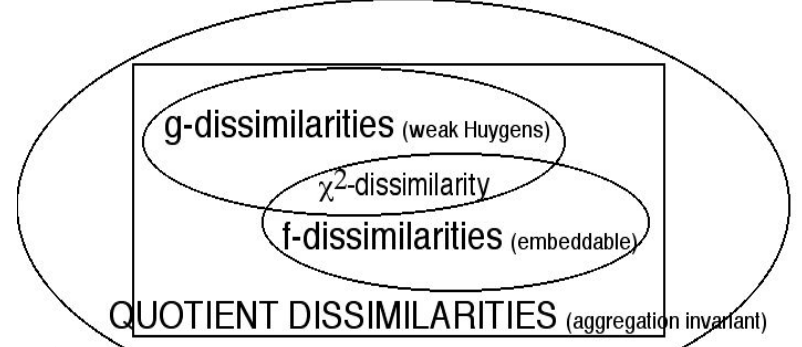

Figure 1. Types of categorical dissimilarities under consideration

Classical factorial correspondence analysis (FCA) consists in visualizing the rows (and columns) of the contingency table in a low-dimensional space, that is in representing row $j$ by factorial coordinates $x_{j \alpha}$ (respectively $y_{k \alpha}$ ) such that $D_{j j^{\prime}}^{\chi}=\sum_{\alpha}\left(x_{j \alpha}-x_{j^{\prime} \alpha}\right)^{2}$; the first dimensions $\alpha=1,2 \ldots$ are chosen to express a maximum proportion of the central inertia ${ }^{3} I_{1}=I_{2}=\frac{1}{n} \chi^{2}$. Here $\chi^{2}$ is the chi-square associated to the contingency table, measuring the total rows/columns dependence, and reconstructed by the sum $\sum_{\alpha=1}^{m} \lambda_{\alpha}$ (with $m=$ $\min (J-1, K-1))$ of the eigenvalues of the associated spectral decomposition problem.

The same eigen-structure is also well-known to arise as the solution to a principal component analysis (PCA) applied on a particular variancecovariance $\left(m_{2} \times m_{2}\right)$ matrix $\Gamma$ among $m_{2}$ variables represented by the columns, with $\operatorname{Tr}(\Gamma)=I_{1}$.

In this paper, we propose a generalized factor analysis for contingency tables -the non linear principal components analysis (NPA)- applicable to any quotient dissimilarity. As a factor-analytic method, NPA determines the projection (hyper-)planes maximizing the explained inertia, but choices must first be made, since $I_{1}, I_{2}, I_{1}^{*}{ }^{4}$ or $I_{2}^{*}$ are all candidates for defining inertia. As a matter of fact, $I_{1}=I_{1}^{*}$ for quotient dissimilarities ${ }^{5}$, while $I_{2}=I_{2}^{*}\left(=I_{1}=I_{1}^{*}\right)$

${ }^{1} g$ is the average profile with associated quotient profile $g_{k} \equiv 1$ (see Equation 1 ).

2 one cannot rule out a priori the possibility of discovering, besides the already identified $g$-and $f$-dissimilarities, a new family $F\left(q, q^{\prime}\right)$ entailing both properties.

${ }^{3}$ by contrast, classical MDS seeks maximizing the low-dimensional representation of $\sum_{j} D_{j g}$, the total unweighted dispersion around $g$.

${ }^{4}$ starred notations denote dual quantities obtained by rows-columns transposition; for instance, $n_{k j}^{*}=n_{j k}$ and $q_{k j}^{*}=q_{j k}$.

${ }^{5} I_{1}^{*}=\sum_{k} \rho_{k} D_{k g^{*}}^{*}=\sum_{k} \rho_{k} \sum_{j} \rho_{j}^{*} F\left(q_{k j}^{*}, 1\right)=\sum_{j} \rho_{j}^{*} \sum_{k} \rho_{k} F\left(q_{j k}, 1\right)=I_{1}$ 
for $g$-dissimilarities. In general, Huygens's weak principle is violated, which can be measured by the eccentricity $\epsilon$ and the dual eccentricity $\epsilon^{*}$ we define as $\epsilon:=\left(I_{1}-I_{2}\right) / I_{2}=I_{1} / I_{2}-1$ and $\epsilon^{*}:=\left(I_{1}^{*}-I_{2}^{*}\right) / I_{2}^{*}=I_{1} / I_{2}^{*}-1$. In general, $\epsilon^{*} \neq \epsilon$. By construction, $\epsilon^{*}=\epsilon=0$ for $g$-dissimilarities, while $\epsilon \geq 0$ and $\epsilon^{*} \geq 0$ for $f$-dissimilarities (see Section 3 ).

To preserve the rows-columns symmetry, we make the choice of defining NPA as a low-dimensional projection of the central inertia $I_{1}$. The associated spectral decomposition yields factor-variables correlations or loadings representing the rows (and the columns) in the unit hypersphere (see Section 2). Moreover, when restricted to $f$-dissimilarities, the same procedure yields rows (and columns) coordinates defined by coordinates $=$ loadings $\times$ distance to the origin (see Equation 8 below), whose squared Euclidean distances restore the (embeddable) dissimilarities $D_{j j^{\prime}}$, as expected. We call this procedure non linear factorial correspondence analysis (NCA). Most of the usual properties of ordinary FCA are still valid, with a notable exception, matching the non-vanishing of the eccentricities: the weighted average of rows or column coordinates is generally not zero anymore (see Section 3).

The search for exotic, non chi-square dissimilarities but yet endowed with attractive formal properties possesses a long tradition in the classification and data analysis community; let us mention the Hellinger dissimilarity investigated by Escoffier (1978) (defined in our set-up as the $f$ dissimilarity $F\left(q, q^{\prime}\right)=\left(\sqrt{q}-\sqrt{q^{\prime}}\right)^{2}$ ), or the logarithmic dissimilarity (with $\left.F\left(q, q^{\prime}\right)=\left(\ln q-\ln q^{\prime}\right)^{2}\right)$ occurring in the statistical analysis of compositional data (Aitchison and Greenacre (2002)): non-chi square dissimilarities $F\left(q, q^{\prime}\right) \neq\left(q-q^{\prime}\right)^{2}$ allow to distort and modulate the contributions of profiles to the global measures of dependence, and to over- or underweight, as finely as wished, the effect of attractions $(q>1)$ and/or repulsions $(q<1)$ (see also Bavaud (2002) for additional examples). To that extent, the construction of factorial decompositions applied to exotic dissimilarities (NPA and NCA) constitutes a natural continuation of this tradition, aimed at visualizing the rows (and columns) in a way corresponding to the chosen distortion.

\section{Non linear PCA for quotient dissimilarities (NPA)}

The goal is to express $I_{1}=I_{1}^{*}$ as the trace of some positive semi-definite (p.s.d.) $(J \times J)$ symmetric matrix $\Gamma$ of components $\gamma_{j j^{\prime}}$, interpretable as

a variance-covariance matrix between variables $\left\{V_{j}\right\}_{j=1}^{J}$ associated to the $J$ rows. Define the $(J \times K)$ matrix $C$ with components

$$
c_{j k}:=\operatorname{sgn}\left(q_{j k}-1\right) \sqrt{\rho_{j}^{*} F\left(q_{j k}, 1\right) \rho_{k}} .
$$

Then, by construction, $\Gamma:=C C^{\prime}$ is p.s.d. It satisfies

$$
\gamma_{j j}=\rho_{j}^{*} \sum_{k} \rho_{k} F\left(q_{j k}, 1\right)=\rho_{j}^{*} D_{j g}
$$


and $\operatorname{Tr}(\Gamma)=I_{1}$. Decreasingly ordered eigenvalues $\lambda_{\alpha}$ and associated eigenvectors $u_{j \alpha}$ (where $\left.\alpha=1, \ldots, m:=\min (J, K)\right)$ are obtained from the spectral decomposition $\Gamma=U \Lambda U^{\prime}$ with $U$ orthogonal and $\Lambda$ diagonal, that is

$\operatorname{cov}\left(V_{j}, V_{j^{\prime}}\right)=\gamma_{j j^{\prime}}=\sum_{\alpha=1}^{m} \lambda_{\alpha} u_{j \alpha} u_{j^{\prime} \alpha} \quad \sum_{\alpha} u_{j \alpha} u_{j^{\prime} \alpha}=\delta_{j j^{\prime}} \quad \sum_{j} u_{j \alpha} u_{j \beta}=\delta_{\alpha \beta}$.

The term $\operatorname{sgn}\left(q_{j k}-1\right)$ in Equation (2) insures that feature $k$ contributes positively to $\operatorname{cov}\left(V_{j}, V_{j^{\prime}}\right)>0$ iff quotients $q_{j k}$ and $q_{j^{\prime} k}$ are either both superior to 1 or both inferior to 1 . By construction, the factors $F_{\alpha}:=\sum_{j} V_{j} u_{j \alpha}$ are uncorrelated: $\operatorname{cov}\left(F_{\alpha}, F_{\beta}\right)=\delta_{\alpha \beta} \lambda_{\alpha}$; moreover,

$$
\operatorname{cov}\left(V_{j}, F_{\alpha}\right)=\lambda_{\alpha} u_{j \alpha} \quad s_{j \alpha}:=\operatorname{corr}\left(V_{j}, F_{\alpha}\right)=\frac{\sqrt{\lambda_{\alpha}}}{\sqrt{\gamma_{j j}}} u_{j \alpha} .
$$

As usual in PCA, the variables $V_{j}$ can be represented by their loadings $s_{j \alpha}$ on the factors $F_{\alpha}$ (see Section 4). Loadings reproduce original correlations in that $\sum_{\alpha} s_{j \alpha} s_{j^{\prime} \alpha}=\operatorname{corr}\left(V_{j}, V_{j^{\prime}}\right)$. Also, the identities $\sum_{\alpha} s_{j \alpha}^{2}=1$ and $\sum_{j} \gamma_{j j} s_{j \alpha}^{2}=\lambda_{\alpha}$ permit defining the contributions of factors $F_{\alpha}$ to the proportion of explained variance of the variables $V_{j}$ (communalities) and vice-versa.

The above NPA is applicable for any quotient dissimilarity $D$ (provided $\gamma_{j j}>0$ for all $j$ ), irrespectively of its possible metric properties. In the chisquare case, $\operatorname{sgn}(q-1) \sqrt{F(q, 1)}=q-1$ and the vector $u_{j m}:=\sqrt{\rho_{j}^{*}}$ is well-known to constitute a normalized eigenvector of $\Gamma$ with associated trivial eigenvalue $\lambda_{m}=0$; this property is false for quotient dissimilarities where $\lambda_{m}>0$ in general.

The spectral decomposition applied on the $(K \times K)$ matrix $\Gamma^{*}:=C^{\prime} C=$ $V \Lambda V^{\prime}$ instead on $\Gamma$ yields a factorial representation of the columns variables $V_{k}^{*}$, with identical eigenvalues $\lambda_{\alpha}$ and with normalized eigenvectors $v_{k \alpha}$ related to normalized eigenvectors $u_{j \alpha}$ of $\Gamma$ by

$$
v_{\alpha}=\frac{1}{\sqrt{\lambda_{\alpha}}} C^{\prime} u_{\alpha} \quad u_{\alpha}=\frac{1}{\sqrt{\lambda_{\alpha}}} C v_{\alpha} .
$$

In summary, matrices $U$ and $V$ (orthogonal) and $\Lambda$ (diagonal) satisfy:

$$
C=U \sqrt{\Lambda} V^{\prime} \quad C^{\prime}=V \sqrt{\Lambda} U^{\prime} \quad \Gamma=C C^{\prime}=U \Lambda U^{\prime} \quad \Gamma^{*}=C^{\prime} C=V \Lambda V^{\prime} .
$$

\section{Non linear FCA for $f$-dissimilarities (NCA)}

The $f$-dissimilarities obey $\operatorname{sgn}\left(q_{j k}-1\right) \sqrt{F\left(q_{j k}, 1\right)}=f\left(q_{j k}\right)-f(1)$. It is convenient to calibrate $f(q)$ (defined up to an affine transformation) so that $f(1)=1$. Moreover, if $f(q)$ is smooth enough, the choice $f^{\prime}(1)=1$ ensures 
$f(q)=q-1+\kappa(q-1)^{2}+0\left((q-1)^{3}\right)$, where $\kappa:=\frac{1}{2} f^{\prime \prime}(1)$ is the reference curvature (measured at the independence value $q=1$ ). One then has

$$
\gamma_{j j^{\prime}}=\sqrt{\rho_{j}^{*} \rho_{j^{\prime}}^{*}} \sum_{k} \rho_{k} f\left(q_{j k}\right) f\left(q_{j^{\prime} k}\right)=\sum_{\alpha=1}^{m} \lambda_{\alpha} u_{j \alpha} u_{j^{\prime} \alpha} .
$$

NCA coordinates $x_{j \alpha}$ for row $j$ (aimed at metrically embedding the categories) are obtained by multiplying the loadings $s_{j \alpha}(4)$ (used to represent the variables in the associated NPA) by the distance to the average profile $\sqrt{D_{j g}}$, that in view of (3) and (4):

$$
x_{j \alpha}:=\sqrt{D_{j g}} s_{j \alpha}=\frac{\sqrt{\gamma_{j j}}}{\sqrt{\rho_{j}^{*}}} s_{j \alpha}=\frac{\sqrt{\lambda_{\alpha}}}{\sqrt{\rho_{j}^{*}}} u_{j \alpha} .
$$

As claimed, and using (7), the $x_{j \alpha}$ are Euclidean coordinates for objects $j \in J$ :

$$
\begin{aligned}
& \sum_{\alpha}\left(x_{j \alpha}-x_{j^{\prime} \alpha}\right)^{2}=\sum_{\alpha=1}^{m} \lambda_{\alpha}\left[\frac{u_{j \alpha}^{2}}{\rho_{j}^{*}}+\frac{u_{j^{\prime} \alpha}^{2}}{\rho_{j^{\prime}}^{*}}-2 \frac{u_{j \alpha} u_{j^{\prime} \alpha}}{\sqrt{\rho_{j}^{*} \rho_{j^{\prime}}^{*}}}\right]=\frac{\gamma_{j j}}{\rho_{j}^{*}}+\frac{\gamma_{j^{\prime} j^{\prime}}}{\rho_{j^{\prime}}^{*}}-2 \frac{\gamma_{j j^{\prime}}}{\sqrt{\rho_{j}^{*} \rho_{j^{\prime}}^{*}}}= \\
= & \sum_{k} \rho_{k}\left(f^{2}\left(q_{j k}\right)+f^{2}\left(q_{j^{\prime} k}\right)-2 f\left(q_{j k}\right) f\left(q_{j^{\prime} k}\right)\right)=\sum_{k} \rho_{k}\left(f\left(q_{j k}\right)-f\left(q_{j^{\prime} k}\right)\right)^{2}=D_{j j^{\prime}} .
\end{aligned}
$$

Proceeding analogously with columns leads to representing feature $k$ in dimension $\alpha$ by the coordinate $y_{k \alpha}$ defined as $y_{k \alpha}:=\frac{\sqrt{\lambda_{\alpha}}}{\sqrt{\rho_{k}}} v_{k \alpha}=\frac{\sqrt{\gamma_{k k}^{*}}}{\sqrt{\rho_{k}}} s_{k \alpha}^{*}=$ $\sqrt{D_{k \rho^{*}}^{*}} s_{k \alpha}^{*}$, where $s_{k \alpha}^{*}=\operatorname{corr}\left(V_{k}^{*}, F_{k}^{*}\right)$ is the corresponding column loading. Using (5) yields the transition formulas

$$
\left\{\begin{array}{l}
y_{k \alpha}=\frac{1}{\sqrt{\lambda_{\alpha}}} \sum_{j \in J} \rho_{j}^{*} f\left(q_{j k}\right) x_{j \alpha} \\
x_{j \alpha}=\frac{1}{\sqrt{\lambda_{\alpha}}} \sum_{k \in K} \rho_{k} f\left(q_{j k}\right) y_{k \alpha} .
\end{array} \quad\right. \text { (transition formulas) }
$$

High- versus low-dimensional coordinates: formula $\sum_{k} \rho_{k}\left(f\left(q_{j k}\right)-\right.$ $\left.f\left(q_{j^{\prime} k}\right)\right)^{2}=D_{j j^{\prime}}$ shows categories $j=1, \ldots, J$ to be metrically embedded by the coordinates $x_{j \alpha}$ (low-dimensional, factorial embedding) or equivalently by the coordinates $\tilde{x}_{j k}:=\sqrt{\rho_{k}} f\left(q_{j k}\right)$ (high-dimensional, original embedding). The two systems of coordinates are linearly related by

$$
x_{j \alpha}=\sum_{k} \sqrt{\rho_{k}} \tilde{x}_{j k} \quad \tilde{x}_{j k}=\sum_{\alpha} \sqrt{\rho_{k}} x_{j \alpha}
$$

that is, $X=\tilde{X} V$, where $V$ is a rotation ${ }^{6}$. Similarly, factorial column coordinates $Y=\left(y_{k \alpha}\right)$ are related to original column coordinates $\tilde{Y}=\left(\tilde{y}_{k j}\right)$ (with $\left.\tilde{y}_{k j}:=\sqrt{\rho_{j}^{*}} f\left(q_{j k}\right)\right)$ as $Y=\tilde{Y} U$ and $\tilde{Y}=Y U^{\prime}$.

${ }^{6}$ Proof: denoting by $\Phi$ the $(J \times J)$ diagonal matrix containing the $\rho_{j}^{*}$, one has $X \stackrel{(a)}{=} \Phi^{-1} U \sqrt{\Lambda} \stackrel{(b)}{=} \Phi^{-1} C V \stackrel{(c)}{=} \tilde{X} V$, where (a) follows from (8), (b) from (6), and (c) follows from the definition $\tilde{X}=\Phi^{-1} C\left(\right.$ since $\left.c_{j k}=\sqrt{\rho_{j}^{*} \rho_{k}} f\left(q_{j k}\right)=\sqrt{\rho_{j}^{*}} \tilde{x}_{j k}\right)$. 
As an application, consider a supplementary row $a$ with quotient profile $a_{k} \geq 0$ obeying $\sum_{k} \rho_{k} a_{k}=1$. Its factorial coordinates $x_{a \alpha}$ can be obtained from its original coordinates $\sqrt{\rho_{k}} f\left(a_{k}\right)$ by transformation (10), yielding $x_{a \alpha}=$ $\sum_{k} \sqrt{\rho_{k}} f\left(a_{k}\right) v_{k \alpha}$ and obeying $\sum_{\alpha}\left(x_{j \alpha}-x_{a \alpha}\right)^{2}=\sum_{k} \rho_{k}\left(f\left(q_{j k}\right)-f\left(a_{k}\right)\right)^{2}=$ $D_{j a}$ by construction. In particular, the factorial coordinates of the average profile $g$ with $g_{k}=1 \forall k$ are $x_{g \alpha}=0$, since $f(1)=0$ : as in usual CA, the average profile is represented at the origin.

Eccentricity: while the coordinates $x_{g \alpha}$ of the average profile $g$ are zero, the average components of the row coordinates $\bar{x}_{\alpha}:=\sum_{j} \rho_{j}^{*} x_{j \alpha}$ are not zero in general: the square of its norm is instead

$$
\sum_{\alpha} \bar{x}_{\alpha}^{2}=\sum_{k} \rho_{k} \bar{f}_{k}^{2} \quad \text { where } \quad \bar{f}_{k}:=\sum_{j} \rho_{j}^{*} f\left(q_{j k}\right) .
$$

The latter is directly related to the eccentricity $\epsilon$ (see Section 1), measuring the violation of Huygens's weak principle:

$$
\begin{array}{r}
I_{1}-I_{2}=\sum_{j} \rho_{j}^{*} D_{j g}-\frac{1}{2} \sum_{j j^{\prime}} \rho_{j}^{*} \rho_{j^{\prime}}^{*} D_{j j^{\prime}} \\
=\sum_{j k} \rho_{j}^{*} \rho_{k} f^{2}\left(q_{j k}\right)-\frac{1}{2} \sum_{j j^{\prime} k} \rho_{j}^{*} \rho_{j^{\prime}}^{*} \rho_{k}\left(f\left(q_{j k}\right)-f\left(q_{j^{\prime} k}\right)\right)^{2} \\
=\sum_{j j^{\prime} k} \rho_{j}^{*} \rho_{j^{\prime}}^{*} \rho_{k} f\left(q_{j k}\right) f\left(q_{j^{\prime} k}\right)=\sum_{k} \rho_{k} \bar{f}_{k}^{2} .
\end{array}
$$

In first approximation, eccentricities behave as $\epsilon \cong c \kappa^{2}$ and $\epsilon^{*} \cong c^{*} \kappa^{2}$ (where $\kappa:=\frac{1}{2} f^{\prime \prime}(1)$ is the reference curvature and $c$ and $c^{*}$ two constants), and thus constitute a measure of distortion between the "exotic profile" $f(q)$ and the "classical profile" $f^{\chi}(q)=q-1$.

\section{An example}

\begin{tabular}{|c||c|c|c|c||c|c|}
\hline hair color $k \rightarrow$ & black & brunette & red & blond & total & $\rho^{*}$ \\
\cline { 1 - 5 } eye color $j \downarrow$ & $\mathrm{W}$ & $\mathrm{X}$ & $\mathrm{Y}$ & $\mathrm{Z}$ & & \\
\hline \hline $\mathrm{A}$ = brown & 68 & 119 & 26 & 7 & 220 & .37 \\
$\mathrm{~B}=$ hazel & 15 & 54 & 14 & 10 & 93 & .16 \\
$\mathrm{C}=$ green & 5 & 29 & 14 & 16 & 64 & .11 \\
$\mathrm{D}=$ blue & 20 & 84 & 17 & 94 & 215 & .36 \\
\hline \hline total & 108 & 286 & 71 & 127 & 592 & 1 \\
\hline$\rho$ & .18 & .48 & .12 & .22 & 1 & \\
\hline
\end{tabular}

\begin{tabular}{|c||c|c|c|c|}
\hline hair color $k \rightarrow$ & $\mathrm{W}$ & $\mathrm{X}$ & $\mathrm{Y}$ & $\mathrm{Z}$ \\
\cline { 1 - 3 } eye color $j \downarrow$ & & & & \\
\hline \hline $\mathrm{A}$ & 1.69 & 1.12 & .99 & .15 \\
$\mathrm{~B}$ & .88 & 1.20 & 1.26 & .50 \\
$\mathrm{C}$ & .43 & .94 & 1.82 & 1.17 \\
$\mathrm{D}$ & .51 & .81 & .66 & 2.04 \\
\hline
\end{tabular}

Table 1: cross counts $n_{j k}$ of eye and hair color of 592 subjects (left), with associated quotients $q_{j k}$ (right). Source: Snee (1974).

Consider the power dissimilarity $f_{\beta}:=\frac{1}{\beta}\left(q^{\beta}-1\right.$ ) (with $\beta>0$ ), such that $f(1)=0, f^{\prime}(1)=1$ and $\kappa:=\frac{1}{2} f^{\prime \prime}(1)=\frac{1}{2}(\beta-1)$. The case $\beta=1$ yields the chi-square dissimilarity; $\beta=0.5$ yields the Hellinger dissimilarity. Table 
2 gives the matrices of squared distance between rows $D_{\beta}$ and columns $D_{\beta}^{*}$ for the three cases $\beta=1, \beta=3$ and $\beta=0.2$, associated with the $(4 \times 4)$ contingency table of table 1 .

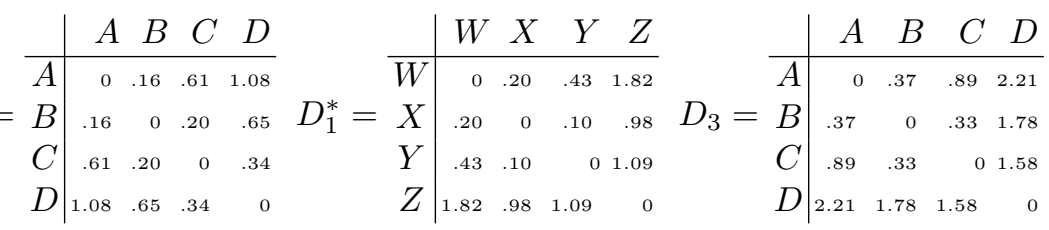

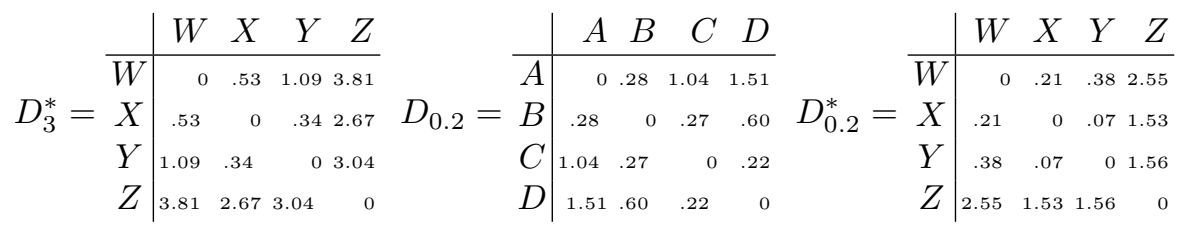

Table 2: squared distances for the power dissimilarity between rows and between columns, for $\beta=1, \beta=3$ and $\beta=0.2$.

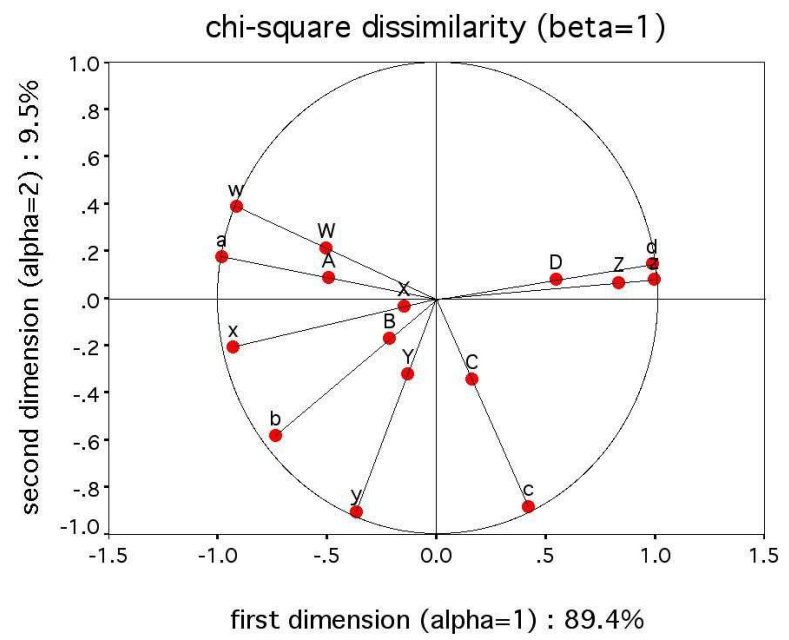

Figure 2. Usual NPA and NCA, with $I_{1}=.23$ and eccentricities $\epsilon=\epsilon^{*}=0$.

Figures 2 to 4 depict the simultaneous visualization of loadings $s_{j \alpha}$ and $s_{k \alpha}^{*}$ (lower case letters: NPA) lying inside the circle of unit radius, and coordinates $x_{j \alpha}$ and $x_{k \alpha}^{*}$ (UPPER CASE LETTERS: NCA), reproducing the distances of table 2 . Loadings and coordinates are not on the same scale: for instance, the transformation $f(q) \rightarrow a f(q)$ entails $x_{j \alpha} \rightarrow a x_{j \alpha}$ but leaves $s_{j \alpha}$ unchanged; however, the figures clearly demonstrate the common factorial structure associated to both NPA and NCA, making $x_{j}$ and $s_{j}$ (as well as $x_{k}^{*}$ and $s_{k}^{*}$ ) parallel. The proportion of explained variance associated to dimension $\alpha$ is $\lambda_{\alpha} / I_{1}$ 


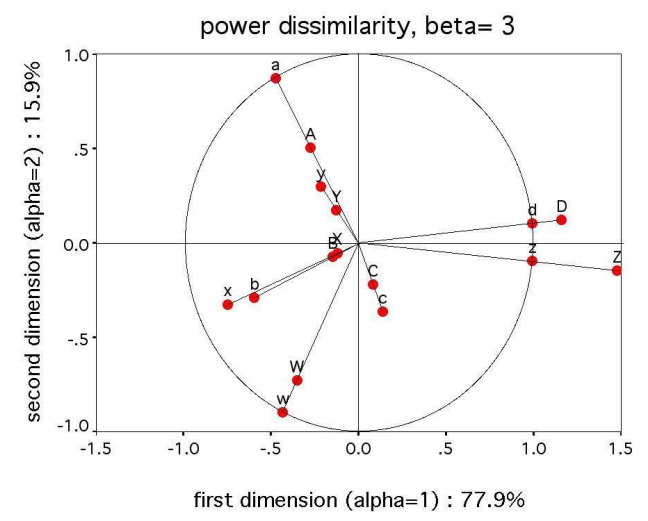

Figure 3. Power dissimilarities with $\beta>1$ increase the contributions of large quotients, namely $q_{D Z}=2.04$. One gets $I_{1}=.67, \epsilon=.28$ and $\epsilon^{*}=.13$.

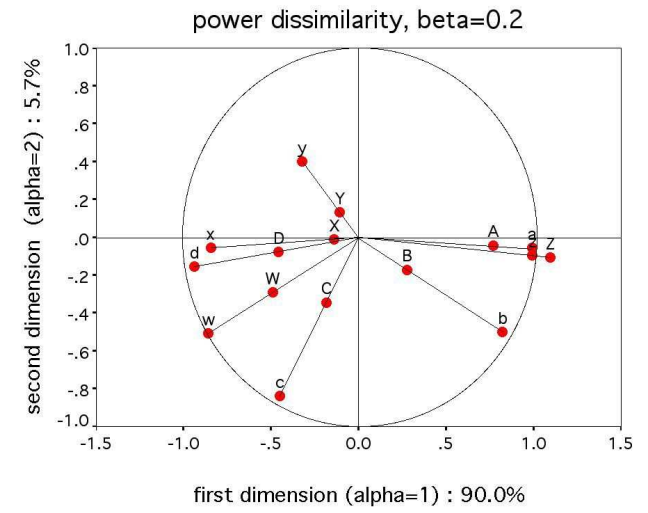

Figure 4. Power dissimilarities with $\beta<1$ increase the contributions of small quotients, namely $q_{A Z}=0.15$. One gets $I_{1}=.35, \epsilon=.12$ and $\epsilon^{*}=.05$.

\section{References}

AITCHISON, J and GREENACRE, M. (2002): Biplots for compositional data, Applied Statistics 51, pp. 375-382

BAVAUD, F. (2002): Quotient Dissimilarities, Euclidean Embeddability, and Huygens' Weak Principle In Jajuga,K. and al. (Eds.): Classification, Clustering and Data Analysis Springer, New York, pp. 195-202

ESCOFIER, B. (1978): Analyse factorielle et distances répondant au principe d'équivalence distributionnelle. Revue de Statistique Appliquée, 26, pp. 29-37 SNEE, R.D. (1974): Graphical Display of Two-Way Contingency Tables,

The American Statistician 28, pp. 9-12 